\title{
Activated $\beta_{2}$ Integrins Restrict Neutrophil Recruitment during Murine Acute Pseudomonal Pneumonia
}

\author{
Zachary S. Wilson ${ }^{1,2}$, Lawrence B. Ahn ${ }^{1}$, William S. Serratelli ${ }^{1}$, Matthew D. Belley ${ }^{3,4,5}$, Joanne Lomas-Neira ${ }^{1}$, \\ Mehmet Sen ${ }^{6}$, and Craig T. Lefort ${ }^{1,3}$ \\ ${ }^{1}$ Division of Surgical Research, Department of Surgery, and ${ }^{4}$ Department of Radiation Oncology, Rhode Island Hospital, \\ Providence, Rhode Island; ${ }^{2}$ Graduate Program in Pathobiology and ${ }^{3}$ Warren Alpert Medical School, Brown University, Providence, \\ Rhode Island; ${ }^{5}$ Department of Physics, University of Rhode Island, Kingston, Rhode Island; and ${ }^{6}$ Department of Biology and \\ Biochemistry, University of Houston, Houston, Texas
}

ORCID ID: 0000-0003-3100-877X (C.T.L.).

\begin{abstract}
Rapid neutrophil recruitment is critical for the efficient clearance of bacterial pathogens from the lungs. Although $\beta_{2}$ integrins and their activation are required for neutrophil recruitment from postcapillary venules of the systemic circulation into inflamed tissues, the involvement of integrins in neutrophil recruitment in response to respiratory infection varies among bacterial pathogens. For stimuli eliciting $\beta_{2}$ integrin-dependent neutrophil influx, including Pseudomonas aeruginosa, it remains unclear whether the activation of $\beta_{2}$ integrins is an essential step in this process. In the current study, we analyze neutrophil trafficking within the lungs of mice infected with Pseudomonas aeruginosa and evaluate the role of $\beta_{2}$ integrin activation through genetic deletion of talin-1 or Kindlin-3 or by pharmacological inhibition of high-affinity $\beta_{2}$ integrins using a small molecule allosteric antagonist. We observe that attenuation of high-affinity $\beta_{2}$ integrins leads to an enhancement of neutrophil emigration into lung interstitium and airspaces. Neutrophil effector functions, including the production of reactive oxygen species and the
\end{abstract}

phagocytosis of bacteria, are only partially dependent on highaffinity $\beta_{2}$ integrins. These results reveal a mechanism by which activated $\beta_{2}$ integrins limit neutrophil entry into the lung tissue and airspaces during acute pseudomonal pneumonia and suggest potential strategies for modulating neutrophil-mediated host defense.

Keywords: neutrophils; pseudomonal pneumonia; Pseudomonas aeruginosa; adhesion; integrin activation

\section{Clinical Relevance}

Neutrophils are essential for host defense against respiratory infection but can also induce tissue damage associated with disease. Our work provides mechanistic insight into the recruitment of neutrophils into the lung tissue and airspaces in response to Pseudomonas aeruginosa, thus informing potential strategies to modulate this process during disease.
Acute respiratory infections remain a significant public health burden, one that is expected to grow with the continued emergence of drug-resistant pathogens. Neutrophil recruitment is a critical early event in innate host defense against bacteria. Bacterial and fungal infections are more frequent and more severe under conditions in which neutrophil recruitment is reduced, such as neutropenia associated with cancer treatments (1). In this context, promoting an influx of neutrophils into infected lung airspaces represents a potential immunomodulatory therapy to complement antibiotic approaches.

(Received in original form July 11, 2016; accepted in final form February 3, 2017)

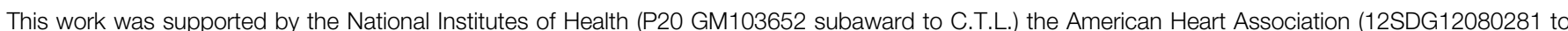
C.T.L.), and the National Research University Fund from the State of Texas (M.S.).

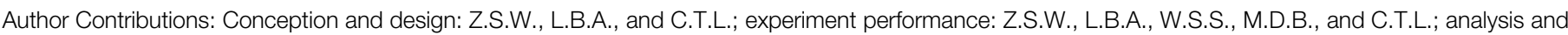
interpretation: Z.S.W., L.B.A., J.L.-N., and C.T.L.; reagent development: W.S.S. and M.S.; and writing and revising the manuscript: Z.S.W. and C.T.L.

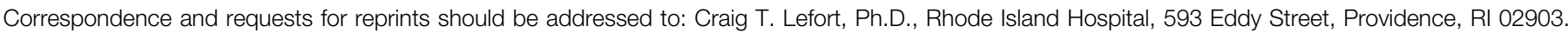
E-mail: craig_lefort@brown.edu

This article has an online supplement, which is accessible from this issue's table of contents at www.atsjournals.org

Am J Respir Cell Mol Biol Vol 56, Iss 5, pp 620-627, May 2017

Copyright (C) 2017 by the American Thoracic Society

Originally Published in Press as DOI: 10.1165/rcmb.2016-0215OC on February 3, 2017

Internet address: www.atsjournals.org 
In the canonical neutrophil recruitment cascade that underlies trafficking from postcapillary venules of the systemic circulation, selectin ligands and integrins expressed on the neutrophil surface mediate rolling and adhesive interactions with inflamed endothelia that precede migration into tissue (2). In the context of respiratory infection, neutrophil recruitment into the lung airspaces occurs primarily from the extensive alveolar capillary network (3-5). The diameter of the neutrophil exceeds that of most alveolar capillary segments, necessitating neutrophil deformation for passage (6). Under homeostatic conditions, neutrophils and other leukocyte populations are highly enriched in the pulmonary circulation because of the geometric constraints that prolong leukocyte transit time compared with that of erythrocytes (7). Sequestration and further concentration of neutrophils within alveolar capillaries in response to inflammatory stimuli occurs as a result of reduced deformability of activated neutrophils in a manner that does not depend on integrin-mediated adhesion $(8,9)$. However, $\beta_{2}$ integrins do play a role in the retention of sequestered neutrophils (10) and in their recruitment into the bronchoalveolar airspaces in response to certain stimuli, including Pseudomonas aeruginosa $(11,12)$. Thus, although the activation of $\beta_{2}$ integrins is obligatory for neutrophil recruitment from the systemic circulation $(13,14)$, it remains unclear whether this mechanism applies to neutrophil recruitment from the pulmonary vasculature.

Integrin affinity for ligand is regulated in an "inside-out" manner by intracellular signal transduction that triggers the binding of talin and Kindlin family proteins to the short cytosolic tail of integrin $\beta$ subunits. talin-1 and Kindlin-3 associate with distinct binding motifs in the $\beta_{2}$ integrin tail and induce structural changes that are transmitted through the transmembrane domains to the extracellular legs and headpiece, ultimately altering the ligandbinding affinity of the headpiece (15). We found previously that talin-1 is responsible for the conversion of the extracellular domain of the $\beta_{2}$ integrin family member lymphocyte function-associated antigen 1 (LFA-1) from a bent to an extended form, whereas Kindlin-3 is needed only for the transition from an intermediate-affinity extended (closed headpiece) state to the high-affinity state with an open headpiece (14). In the absence of Kindlin-3, neutrophils retain certain adhesive functions mediated by partially active $\beta_{2}$ integrins (14). In a similar manner, $\alpha / \beta$ I-like allosteric antagonists, including the small molecule XVA143, prevent the $\beta_{2}$ integrin headpiece from opening to reach the high-affinity state, but permit the partially active conformer (16-18).

In this study, we evaluated the requirement for $\beta_{2}$ integrin activation in neutrophil recruitment during acute respiratory infection of mice with $P$. aeruginosa. We demonstrate that blocking the activation of $\beta_{2}$ integrins, either genetically or pharmacologically, promotes neutrophil emigration into the lung interstitium and airspaces.

\section{Materials and Methods}

Additional description of the materials and methods is provided in the online supplement.

\section{Animals}

All animal studies were approved by the Lifespan Animal Welfare Committee. Mice were housed in a specific pathogen-free facility at Rhode Island Hospital. Mice harboring floxed $T \ln 1$ alleles (19), floxed Fermt3 alleles (20), and the Mx1-Cre transgene (21) have been described. To generate mixed chimeras, 8 - to 12-week-old C57BL/6 mice (Jackson Laboratory, Bar Harbor, ME) were lethally irradiated (10 Gy, single dose) and then reconstituted by intravenous injection of bone marrow cells from two sources at a 1:1 ratio: a wild-type mouse expressing enhanced green fluorescent protein (EGFP) under the ubiquitin $\mathrm{C}$ (UbC) promoter, and either a $\operatorname{Tln} 1^{\text {flox/flox }} \mathrm{Mx} 1$-Cre or a Fermt $3^{\text {llox/flox }} \mathrm{Mx1-Cre}$ mouse. Deletion of the gene encoding talin-1 or Kindlin-3 was induced by intraperitoneal injection of $250 \mu \mathrm{g}$ of polyinosinic-polycytidylic acid (InvivoGen, San Diego, CA), three doses, each 2 days apart, starting 4 weeks after hematopoietic reconstitution, inducing near complete loss of the respective protein in neutrophils (14). Mice were used for experiments 4-8 weeks after polyinosinic-polycytidylic acid administration.

\section{Acute Respiratory Infection}

Prior to infection, a sample of peripheral blood was collected by saphenous venipuncture. The cytotoxic $P$. aeruginosa strain PA103 (22) was grown on solid media before culturing to log phase in Luria broth. Bacteria were suspended in sterile $0.9 \%$ saline, and the number of colonyforming units (CFUs) was determined by a standard curve according to optical density at $540 \mathrm{~nm}$. Under vaporized isoflurane anesthesia, a $50-\mu l$ bolus of $1 \times 10^{6} \mathrm{CFU}$ $P$. aeruginosa was administered to mice by intratracheal inhalation. For some experiments, intravenous injection of the blocking anti-CD18 antibody (30 $\mu \mathrm{g}$, clone GAME-46), anti-intercellular adhesion molecule 1 (ICAM-1) antibody $(30 \mu \mathrm{g}$, clone YN1), the $\beta_{2}$ integrin allosteric antagonist XVA143 (16) (3 $\mu$ l of $10 \mathrm{mM}$ XVA143 in $0.1 \mathrm{ml}$ saline), or vehicle (dimethyl sulfoxide) immediately preceded infection. The mice were monitored closely for signs of morbidity and mortality.

\section{Lung Fractionation}

Methods to distinguish the intravascular and interstitial cell populations within lung tissue have been described (23). Briefly, at 4 hours after infection, the mice were anesthetized and then given $1 \mu \mathrm{g}$ phycoerytherin (PE)-conjugated anti-Ly6G (BioLegend) by intravenous injection. Five minutes later, the pulmonary circulation was perfused with $10 \mathrm{ml}$ Hanks Balanced Salt Solution (containing $\mathrm{Ca}^{2+} / \mathrm{Mg}^{2+}$ ) at physiological pressure $\left(25 \mathrm{~cm} \mathrm{H}_{2} \mathrm{O}\right)$ into the beating right ventricle, allowing exsanguination through a left-ventricle incision. The bronchoalveolar lavage (BAL) was collected by repeated (at least five times) infusion-withdrawal of $1 \mathrm{ml}$ of phosphate-buffered saline containing $1 \mathrm{mM}$ ethylenediaminetetraacetic acid and $0.5 \%$ bovine serum albumin. Lungs were harvested and then digested using an enzyme cocktail (Miltenyi, San Diego, CA) Digested tissue and BAL samples were labeled with allophycocyanin (APC)conjugated anti-Ly6B2 (Miltenyi) to identify all neutrophils by flow cytometry on a MACSQuant instrument (Miltenyi). In mixed chimeric mice, wild-type and gene-deficient neutrophils were delineated according to expression of EGFP. The number of $P$. aeruginosa CFUs in the BAL was determined by plating serial dilutions on solid media.

\section{Statistical Analysis}

For comparison between two groups, twotailed Student's $t$ tests were performed. For comparison among more than two groups, 
one-way analysis of variance and Tukey's post hoc test was used. For comparison of adjacent compartments (blood, intravascular, interstitial, BAL) within mixed chimeric mice, paired analyses were performed. $P$ values $<0.05$ were considered significant. All analyses were performed using Prism GraphPad software.

\section{Results}

\section{Integrin Activation Regulates Neutrophil Recruitment in Response to $P$. Aeruginosa}

$\beta_{2}$ integrins play a role in neutrophil influx into bronchoalveolar airspaces in response to acute respiratory infection with $P$. aeruginosa $(11,12)$. We confirmed this previous finding by observing a significant reduction in recruited neutrophils in the BAL of mice receiving a blocking antibody against $\beta_{2}$ integrins (anti-CD18, clone GAME-46) immediately prior to infection (Figure 1A). There is conflicting prior evidence of a role for ICAM-1, the primary endothelial ligand for $\beta_{2}$ integrins, in pulmonary neutrophil recruitment stimulated by $P$. aeruginosa (24). In our hands, antibody blockade of ICAM-1 did not significantly affect neutrophil recruitment (see Figure E1 in the online supplement).

To evaluate the potential involvement of integrin activation in this process, we took an approach using mixed chimeric mice that allows for the competitive in vivo recruitment of wild-type and gene knockout neutrophils in an internally controlled inflammatory environment. We generated two types of mixed chimeric mice that produce both wild-type and either talin-1- or Kindlin-3-deficient leukocytes, distinguished by their expression of EGFP (wild-type leukocytes had a UbC-EGFP transgene that is active in all neutrophils [Figure E2]). In experiments using the mixed chimera format, the frequency of wild-type and knockout neutrophils always sums to $100 \%$ within each compartment (e.g., blood, tissue, airspaces). How the frequency of knockout neutrophils changes along the recruitment pathway therefore indicates a competitive advantage or disadvantage relative to wild-type neutrophils and provides insight into the mechanisms of neutrophil trafficking.

Mixed chimeric mice were infected with $1 \times 10^{6}$ CFUs of $P$. aeruginosa strain

A

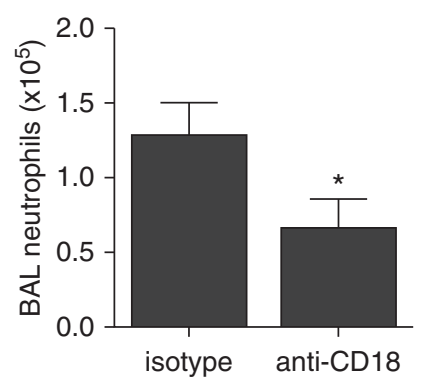

B

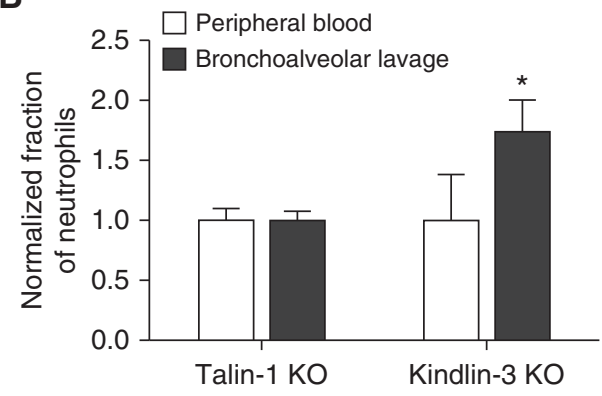

C

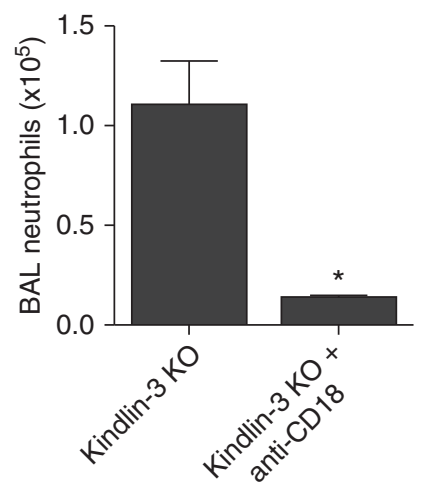

Figure 1. Kindlin-3 deficiency enhances neutrophil recruitment into infected airspaces. (A) Neutrophil influx into the airspaces as measured by neutrophil number in the BAL 4 hours after intratracheal instillation of $1 \times 10^{6} \mathrm{CFUs}$ of Pseudomonas aeruginosa. Mice received either $30 \mu \mathrm{g}$ isotype or $30 \mu \mathrm{g}$ anti-CD18 (clone GAME-46) intravenously prior to infection $(n=8)$ ). *Significantly different from isotype. $(B)$ In mixed chimeric mice infected with $1 \times 10^{6}$ CFUs of $P$. aeruginosa, the frequency of talin-1

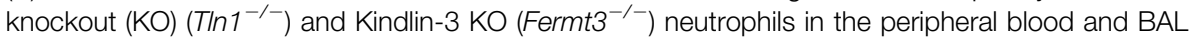
was measured. The data were normalized such that within each experiment, the frequency of KO neutrophils in the peripheral blood was set to 1, and the frequency of $\mathrm{KO}$ neutrophils in the BAL was divided by the frequency in the blood $(n=4)$. * Significantly different from peripheral blood frequency. $(C)$ In mixed chimeric mice with Kindlin-3 KO neutrophils (49.2 $\pm 6.9 \%$ in control, $46.5 \pm$ $3.3 \%$ in anti-CD18 treated), the number of BAL neutrophils was determined 4 hours after infection with $1 \times 10^{6} \mathrm{CFUs}$ of $P$. aeruginosa $(n=3)$. ${ }^{*}$ Significantly different from isotype. All data are expressed as mean \pm SEM.

PA103 by intratracheal inoculation. At 4 hours after infection, we determined the fraction of wild-type and knockout neutrophils that were recruited into the airspaces. Neutrophils lacking talin-1 exhibited a frequency (relative to wild-type neutrophils) in the peripheral blood prior to infection similar to the frequency they exhibited in the BAL of infected mice (Figure 1B), suggesting that talin-1 is not required for neutrophil recruitment during the acute response to $P$. aeruginosa. In contrast, Kindlin-3 knockout neutrophils represented a significantly higher proportion of neutrophils in the BAL than they did in the peripheral blood, indicating that neutrophils lacking Kindlin-3 had a competitive advantage over wild-type neutrophils in their recruitment into the airspaces (Figure 1B). The recruitment of Kindlin-3 knockout neutrophils into the airspaces was still dependent on $\beta_{2}$ integrins, because anti-CD18 pretreatment reduced their numbers in the $\mathrm{BAL}$ (Figure 1C). Together, these results suggest that neutrophils use $\beta_{2}$ integrins to migrate into the airspaces in response to $P$. aeruginosa, but that the talin-1- and Kindlin-3-dependent activation of integrins is not required.

\section{Integrin Activation Restricts Neutrophil Emigration from the Pulmonary Vasculature}

To gain further insight into the role of integrin activation in neutrophil 
recruitment into the airspaces, we analyzed the distribution of neutrophils within the lung tissue. To do so, we implemented an approach (23) in which neutrophils still within the vasculature are labeled by intravenous delivery of PE-anti-Ly6G mAb just prior to the experimental end point. Cells exposed to this circulating label include those marginated or sequestered in the lung but not those that have emigrated into the parenchyma. The pulmonary circulation is then perfused to remove excess label as well as to wash out those blood cells that are not marginated, and then BAL and lung tissue are collected sequentially. Digested lung tissue is labeled with a second neutrophil marker (APC-anti-Ly6B2) to identify all neutrophils. Thus, by flow cytometry, $\mathrm{PE}^{+} \mathrm{APC}^{+}$cells represent intravascular neutrophils, and $\mathrm{PE}^{-} \mathrm{APC}^{+}$cells indicate interstitial neutrophils (Figure 2A). Monocytes, which also express Ly6B2, were present in low numbers in the interstitium at this time point, compared with neutrophils, and were excluded from analyses by their higher Ly6B2 levels and scatter properties (Figure E2).

In the lung tissue of mixed chimeric mice at 4 hours after infection, there was a significant increase in the fraction of talin-1 and Kindlin-3 knockout neutrophils in the interstitium relative to wild-type neutrophils (Figure 2B), indicating a more rapid emigration of neutrophils with defective integrin activation. When viewed in the context of circulating neutrophils, talin-1 knockout neutrophils were less frequent in the lung intravascular compartment than in the peripheral blood prior to infection, whereas Kindlin-3 neutrophils exhibited similar frequencies between these fractions (Figure 2C). Respiratory infection with $P$. aeruginosa significantly reduced the frequency of circulating talin-1 knockout neutrophils relative to wild-type, suggesting that neutrophil release from bone marrow may be defective (Figure 2C). Because the frequency of circulating Kindlin-3 knockout neutrophils was similar to their frequency in lung intravascular fraction, it
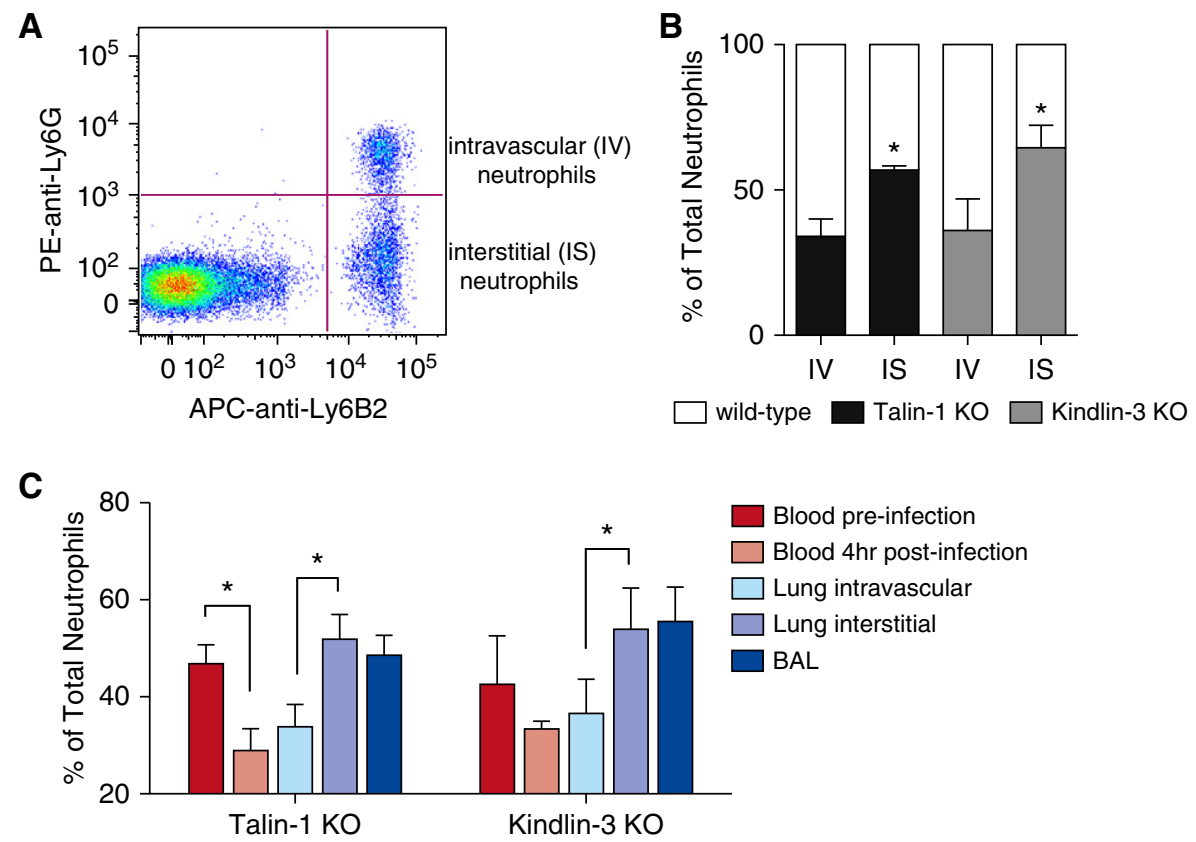

Figure 2. Activated integrins restrict neutrophil emigration. (A) Flow cytometry analysis of intravascular (IV; phycoerytherin [PE] ${ }^{+}$allophycocyanin $[\mathrm{APC}]^{+}$) and interstitial $\left(\mathrm{IS} ; \mathrm{PE}^{-} \mathrm{APC}^{+}\right.$) neutrophil populations within digested lung tissue. Wild-type, talin-1 KO, and Kindlin-3 KO neutrophil frequency in either $(B)$ the lung tissue fraction or $(C)$ the peripheral blood, lung tissue, and BAL fractions, of mixed chimeric mice at 4 hours after infection with $1 \times 10^{6} \mathrm{CFUs}$ of $P$. aeruginosa. In $B$ and $C$, data are expressed as the absolute frequency of the $K O$ neutrophil population within each fraction (the remaining are wild type). Data are expressed as mean \pm SEM ( $n=4$ each). Statistical comparisons were performed between adjacent compartments. In $B$, *significant difference between IS and IV fraction of KO neutrophils. In $C$, *significant difference between indicated adjacent fractions.

suggests that neutrophil sequestration and retention within the pulmonary vasculature may occur independently of Kindlin-3. As seen in the isolated analysis of lung tissue (Figure 2B), side-by-side quantification of all compartments indicates that both talin-1- and Kindlin-3-deficient neutrophils exhibited a competitive advantage over wild-type neutrophils in emigrating into the interstitium (Figure 2C). Together, these results suggest that activated integrins restrict neutrophil emigration from the pulmonary vasculature during acute pseudomonal pneumonia.

\section{Selective Inhibition of High-Affinity $\boldsymbol{\beta}_{2}$ Integrins Modulates Neutrophil Recruitment}

XVA143 is in a class of antagonists that prevent the opening of the headpiece that brings $\beta_{2}$ integrins into their high-affinity state (16). Antagonists in this class do not impair the induction of extended, partially active $\beta_{2}$ integrins, the so-called intermediate-affinity state, but do block neutrophil adhesion mediated by highaffinity $\beta_{2}$ integrins (16-18). We have shown previously that Kindlin-3 is not needed for extension of the extracellular domain to activate LFA-1 to its intermediate-affinity state, but is required for achieving the high-affinity state (14). XVA143 thus mimics Kindlin-3 deficiency in its effects on $\beta_{2}$ integrin structure and neutrophil adhesive function $(14,15)$.

We demonstrated previously that Kindlin-3-deficient neutrophils fail to convert from a rolling to an arrested phenotype in postcapillary venules in response to the chemokine $(\mathrm{C}-\mathrm{X}-\mathrm{C})$ motif ligand 1 (CXCL1) (14). To confirm the in vivo efficacy of XVA143 in blocking neutrophil arrest in postcapillary venules of the systemic circulation, we performed intravital imaging of the mouse cremaster muscle. Surgical exteriorization of the cremaster induces a local inflammatory response and P-selectin-dependent neutrophil rolling in postcapillary venules, with few arrested neutrophils (25). Using this model, we have shown previously that rapid neutrophil arrest in response to intravenous injection of the chemokine CXCL1 is dependent on both talin-1 and Kindlin-3 (14). In the studies performed here, CXCL1-induced neutrophil arrest in postcapillary venules of the cremaster muscle was almost entirely blocked by pretreatment of mice with XVA143, similar 
to the effect of antibody blockade of LFA-1 (Figure 3).

We then assessed the effect of XVA143 on neutrophil recruitment in response to respiratory $P$. aeruginosa infection of C57BL/6 mice. Pretreatment of mice with XVA143 enhanced neutrophil influx into the bronchoalveolar airspaces and promoted emigration from the vasculature into the interstitium (Figure 4A). Thus, XVA143 modulates neutrophil recruitment in the lungs during acute pseudomonal pneumonia in a manner similar to genetic deficiency of Kindlin-3. Because neutrophils can induce bystander tissue injury, we measured the wet-to-dry ratio of the lungs to assess pulmonary edema. At 4 hours after infection, there was a significant increase in the wet-to-dry ratio, but the extent of edema was no different between mice receiving vehicle and those treated with XVA143 (Figure 4B). Histological analyses of lung tissue sections supported these findings (Figure E3). Finally, we measured the clearance of bacteria from lung airspaces by measuring the number of CFUs in the BAL 4 hours after intratracheal administration of $1 \times 10^{6}$ CFU P. aeruginosa. In mice pretreated with either XVA143 or vehicle control, the burden of $P$. aeruginosa was reduced by 30 - to 40 -fold; XVA143 did not significantly affect the clearance of $P$. aeruginosa from the airspaces (Figure 4C).

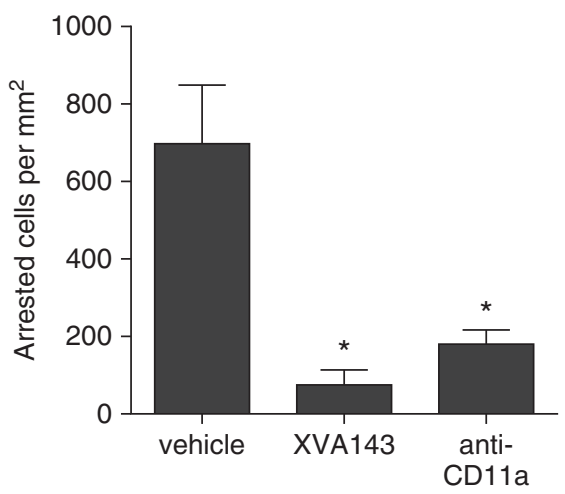

Figure 3. XVA143 blocks neutrophil adhesion in postcapillary venules. C57BL/6 mice were pretreated with either vehicle (DMSO), XVA143, or anti-CD11a (clone M17/4) by intravenous injection. During intravital imaging of the cremaster muscle microvasculature, the number of arrested neutrophils per vessel wall area in a postcapillary venule was determined 30 seconds after intravenous injection of 600 ng chemokine (C-X-C) motif ligand 1 ( $n=3$ per condition). ${ }^{*}$ Significantly different from vehicle.

\section{High-Affinity $\boldsymbol{\beta}_{\mathbf{2}}$ Integrins and Neutrophil Effector Function}

Despite an augmented neutrophil recruitment into airspaces in response to $P$. aeruginosa, XVA143-treated mice cleared bacteria to a similar extent as control mice at the early 4 -hour time point. To address whether high-affinity $\beta_{2}$ integrins are required for neutrophil effector functions that contribute to the eradication of pathogens, we assayed for oxidative burst and phagocytosis of $P$. aeruginosa. Neutrophils used in these assays were derived from conditionally immortalized murine myeloid progenitors that were differentiated in the presence of stem cell factor and granulocyte colony-stimulating factor (26). More than $80 \%$ of cells obtained through this method expressed the neutrophil marker Ly6G (Figure E4). Genetic deletion of talin-1, Kindlin-3, or CD18 (integrin $\beta_{2}$ subunit) in myeloid progenitors was achieved through CRISPRCas9 targeting (Figure E4). Neutrophils lacking talin-1, Kindlin-3, or $\beta_{2}$ integrins, as well as wild-type neutrophils treated with XVA143, retained the capacity to release extracellular superoxide anion in response to $P$. aeruginosa (Figure 5A). These assays were performed in the presence of an antiadhesive surface to specifically isolate the recognition of $P$. aeruginosa as the stimulus of oxidative activity.

Phagocytosis assays were performed using the $\mathrm{pH}$-sensitive pHrodo Green dye conjugated to heat-killed $P$. aeruginosa (PA103-pHrodo), such that only internalized particles gained fluorescence (27). Neutrophil phagocytosis of $P$. aeruginosa was significantly reduced by either talin-1, Kindlin-3, or $\beta_{2}$ integrin deficiency, or by XVA143 treatment (Figure 5B). As a control, neutrophils exposed to PA103-pHrodo at a low temperature were unable to internalize the particles (Figure 5B). Together, these results indicate that high-affinity $\beta_{2}$ integrins play a role in phagocytosis of $P$. aeruginosa but are not required for the adhesionindependent generation of superoxide in response to $P$. aeruginosa.

\section{Discussion}

$\beta_{2}$ integrins represent the dominant neutrophil adhesion receptors, as their loss is the genetic cause of leukocyte adhesion deficiency type I (LAD-I) in humans (28).
LAD-I is characterized by recurrent bacterial and fungal infections. Likewise, leukocyte adhesion deficiency type III resulting from mutation of the FERMT3 gene encoding Kindlin-3 also manifests in a compromised host defense, at least in part because of neutrophil recruitment defects $(13,14,29-31)$. Activation of $\beta_{2}$ integrins by talin- 1 and Kindlin-3 is necessary for neutrophil recruitment from postcapillary venules (14). However, the mechanisms of neutrophil trafficking in the lungs are fundamentally different from those that underlie neutrophil recruitment from other vascular beds throughout the body (32). Here, we have found that in the context of acute respiratory infection of mice with $P$. aeruginosa, neutrophil recruitment into the lung interstitium and bronchoalveolar airspaces is augmented when $\beta_{2}$ integrin activation is blocked. By using a technique to distinguish the neutrophil populations in lung tissue, we specifically identified emigration from the pulmonary vasculature as the primary step that is accelerated by antagonizing the activation of $\beta_{2}$ integrins.

Talins and Kindlins bind directly to the short cytosolic tail of integrin $\beta$ chains and induce changes in integrin structure that regulate affinity for ligand (33). We demonstrated previously that talin- 1 and Kindlin-3 play distinct roles in the process of activating the $\beta_{2}$ integrin LFA-1 (14). Those studies on murine neutrophils found that talin-1 stabilizes the extended form of LFA-1, even in the absence of Kindlin-3, but both proteins are required for neutrophil arrest and recruitment to the inflamed peritoneal cavity (14). In the current study of acute respiratory infection with $P$. aeruginosa, talin-1and Kindlin-3-deficient neutrophils also exhibit phenotypic differences and similarities. When compared with wild-type neutrophils in mixed chimeric mice, Kindlin-3 knockout neutrophil influx into the airspaces was augmented, whereas talin-1 knockout neutrophils showed no overall difference with their wild-type counterpart. This latter outcome may be the additive result of reduced neutrophil release from the bone marrow and/or reduced retention within pulmonary capillaries, both of which counteract the enhanced emigration of talin-1 knockout neutrophils into the interstitium. By contrast, our data indicate that Kindlin-3 deficiency does not significantly affect circulating neutrophils or their sequestration/retention in alveolar 
A

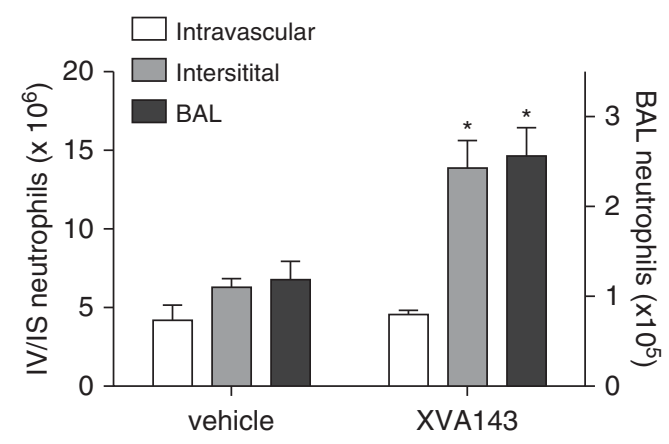

B

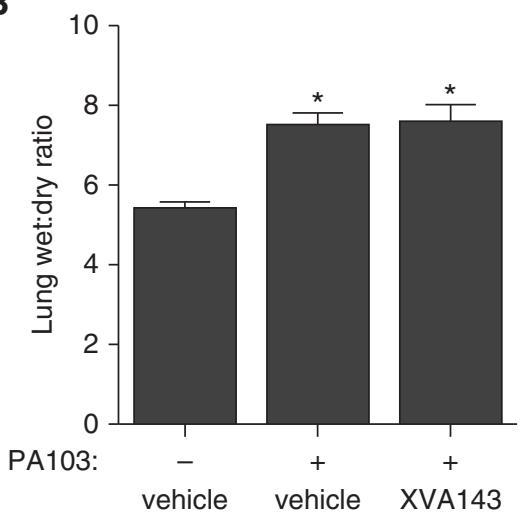

C

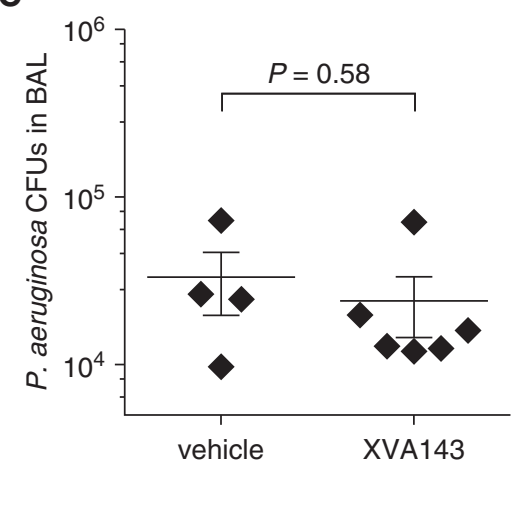

Figure 4. XVA143 promotes neutrophil recruitment into interstitium and airspaces. C57BL/6 mice were pretreated with either vehicle (DMSO) or XVA143 by intravenous injection immediately prior to intratracheal instillation of $1 \times 10^{6} \mathrm{CFUs}$ of $P$. aeruginosa. (A) The number of neutrophils in the lung tissue intravascular and interstitial fractions, and BAL, measured at 4 hours after infection. A representative experiment is shown $(n=3)$. ${ }^{*}$ Significantly different from the same fraction from vehicle control. (B) At 4 hours after infection (control mice received saline), the lungs were harvested and weighed. After drying for 72 hours, the lungs were again weighed, and the wet-to-dry weight ratio was calculated. *Significantly different from uninfected vehicle control. (C) Bacterial load in the BAL at 4 hours after infection.

capillaries, but Kindlin-3-deficient neutrophils exhibit a more robust emigration than do wild-type neutrophils. These results suggest that high-affinity $\beta_{2}$ integrins restrict neutrophil transmigration out of the pulmonary vasculature and into the interstitial spaces, where neutrophils can then cross the epithelium and enter the airspaces in an integrin activation-independent manner. The precise mechanisms of this restriction on neutrophil emigration from the pulmonary circulation remain unclear. Inactivation of $\beta_{2}$ integrins is important for the cyclic adhesion and de-adhesion that is needed for efficient leukocyte motility $(34,35)$. The integrin inactivation process may be a rate-limiting step in neutrophil recruitment in the context of acute pseudomonal pneumonia. Another possibility is that engaged high-affinity $\beta_{2}$ integrins may transduce outside-in signals that attenuate the molecular processes involved in transendothelial migration in the lungs.
Our studies do not directly address the integrin subtype(s) whose activation is involved in regulating neutrophil trafficking during acute pseudomonal pneumonia. Previous work has shown that both of the $\beta_{2}$ integrin subtypes that are highly expressed by neutrophils, LFA-1 and macrophage antigen-1, play a nonredundant role in neutrophil recruitment during the acute response to $P$. aeruginosa (24). Talins and Kindlins are common activators of all integrin subtypes; thus, $\beta 1$ integrin
A

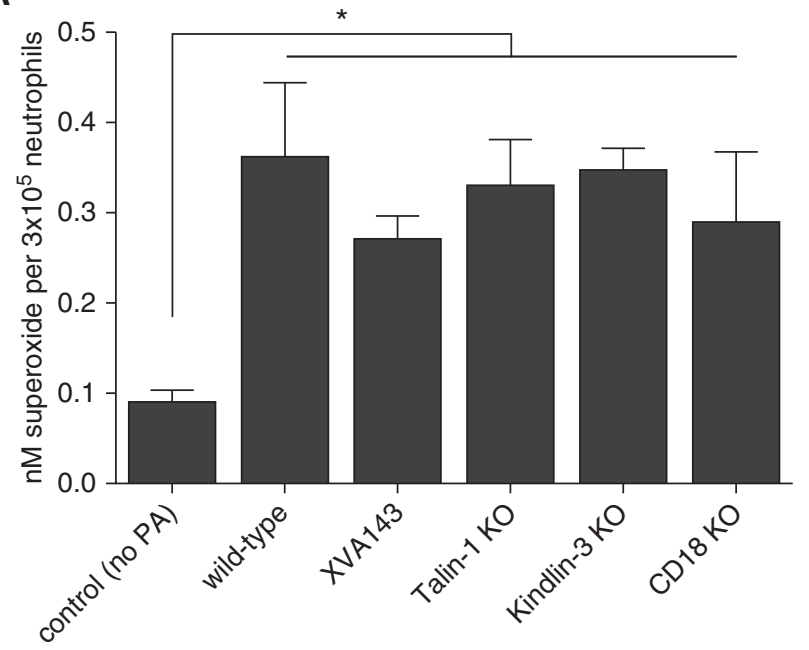

B

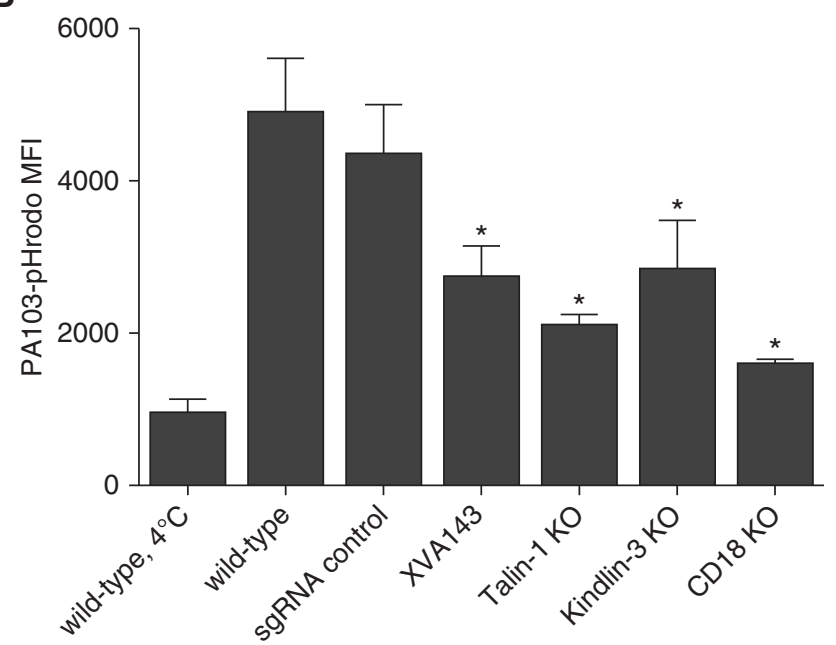

Figure 5. Impaired $\beta_{2}$ integrin activation attenuates neutrophil effector function. (A) Murine neutrophil release of superoxide anion was quantified by measuring the reduction of ferricytochrome $\mathrm{C}$ over the course of 2 hours. Oxidative burst was stimulated by exposing neutrophils to $P$. aeruginosa (PA) $(n=3)$. *Significantly different from control wild-type neutrophils not exposed to PA. (B) Murine neutrophil phagocytosis of heat-killed $P$. aeruginosa conjugated to pHrodo Green dye was measured after 2 hours of exposure at $37^{\circ} \mathrm{C}$ by flow cytometry of Ly6G-expressing cells. Data are expressed as pHrodo Green mean fluorescence intensity $(M F I) \pm \operatorname{SEM}(n=3)$. *Significantly different from wild-type. sgRNA, single guide RNA. 
activation would also be defective in talin-1 and Kindlin-3 knockout neutrophils. XVA143 binds to the $\beta_{2}$ integrin subunit and inhibits the transition of both LFA-1 and macrophage antigen-1 into their highaffinity state (16). The effects of XVA143 on neutrophil recruitment in our study mimic those observed for Kindlin-3 deficiency, which suggests that $\beta_{2}$ integrins, rather than $\beta 1$ integrins, are the receptors that restrict neutrophil emigration from the pulmonary vasculature in this context. Furthermore, although the modulation of neutrophil trafficking and phagocytosis by XVA143 is similar to the phenotype of Kindlin-3 knockout neutrophils, because Kindlin-3 also plays a role in downstream signaling (13), there are likely to be some neutrophil functions that are differentially affected by XVA143 versus Kindlin-3 deficiency.

Neutrophils are essential for host defense against $P$. aeruginosa and other bacterial infections of the lungs $(32,36-38)$. Neutropenic human patients $(39,40)$ and laboratory mice $(36,41,42)$ have enhanced susceptibility to pseudomonal pneumonia. Inefficient rapid, early neutrophil recruitment, even when corrected within 24 hours, resulted in 10 -fold more bacteria in the airspaces at 48 hours in a murine model of pneumococcal pneumonia (43). Potentiating the early phase of neutrophil recruitment may represent a therapeutic approach in the age of antibiotic resistance.
For pseudomonal pneumonia, our studies suggest that high-affinity $\beta_{2}$ integrins are a potential target for such a therapeutic strategy. At the same time, the nonspecific nature of the neutrophil antimicrobial response can induce bystander tissue injury (44). Thus, promoting neutrophil recruitment into lung airspaces may come at an overall cost to the host under certain conditions. In our studies, we did not observe additional injury to the lung in mice receiving XVA143. However, our experiments were focused on the acute response to a relatively large bacterial bolus.

$P$. aeruginosa is a common cause of nosocomial pneumonia, primarily in patients with suppressed immunity (45). Our studies used immunocompetent mice to probe the mechanisms of neutrophil recruitment elicited by $P$. aeruginosa. Despite enhancing neutrophil entry into the airspaces, XVA143 treatment did not significantly affect bacterial clearance. Although it is possible that impaired neutrophil effector function in XVA143treated mice contributed to unimproved host defense, it seems that both control mice and those receiving XVA143 were able to effectively clear $P$. aeruginosa. Further research is required to determine whether neutrophil recruitment during pseudomonal pneumonia in the presence of underlying innate immune dysfunction occurs by mechanisms related to those described here, and whether promoting neutrophil recruitment by inhibiting $\beta_{2}$ integrin activity improves host defense in that context.

In summary, we have shown that blockade of $\beta_{2}$ integrin activation, either through genetic deletion of talin-1 or Kindlin-3, or with the allosteric antagonist XVA143, promotes neutrophil emigration from pulmonary vasculature during acute respiratory infection with $P$. aeruginosa. Nonselective blockade of all $\beta_{2}$ integrins inhibits neutrophil recruitment, suggesting that inactive or partially active $\beta_{2}$ integrins are sufficient to mediate the interactions necessary for entry into the tissue and airspaces. Overall, this study furthers our understanding of neutrophil trafficking in the lungs and provides additional evidence that the mechanisms driving neutrophil recruitment during respiratory infection are fundamentally different from those described for neutrophil recruitment from systemic vascular beds.

Author disclosures are available with the text of this article at www.atsjournals.org.

Acknowledgments: The authors thank Feng Feng, Dr. Brian LeBlanc, and Dr. Xian O'Brien for technical assistance. They also thank Dr. Markus Moser, Dr. Reinhard Fässler, and Dr. David Critchley for providing the mouse strains used in this study and Dr. Paul Ekert for providing the lentiviral/conditional Hoxb8 plasmid.

\section{References}

1. Freifeld AG, Bow EJ, Sepkowitz KA, Boeckh MJ, Ito JI, Mullen CA, Raad II, Rolston KV, Young JA, Wingard JR; Infectious Diseases Society of Americaa. Clinical practice guideline for the use of antimicrobial agents in neutropenic patients with cancer: 2010 update by the Infectious Diseases Society of America. Clin Infect Dis 2011;52:427-431.

2. Ley K, Laudanna C, Cybulsky MI, Nourshargh S. Getting to the site of inflammation: the leukocyte adhesion cascade updated. Nat Rev Immunol 2007;7:678-689.

3. Loosli CG, Baker RF. Acute experimental pneumococcal (type I) pneumonia in the mouse: the migration of leucocytes from the pulmonary capillaries into the alveolar spaces as revealed by the electron microscope. Trans Am Clin Climatol Assoc 1962;74:15-28.

4. Shaw JO. Leukocytes in chemotactic-fragment-induced lung inflammation. Vascular emigration and alveolar surface migration. Am J Pathol 1980;101:283-302.

5. Downey GP, Worthen GS, Henson PM, Hyde DM. Neutrophil sequestration and migration in localized pulmonary inflammation. Capillary localization and migration across the interalveolar septum. Am Rev Respir Dis 1993;147:168-176.

6. Doerschuk CM, Beyers N, Coxson HO, Wiggs B, Hogg JC. Comparison of neutrophil and capillary diameters and their relation to neutrophil sequestration in the lung. J Appl Physiol (1985) 1993; 74:3040-3045.

7. Doerschuk CM. Mechanisms of leukocyte sequestration in inflamed lungs. Microcirculation 2001;8:71-88.
8. Worthen GS, Schwab B III, Elson EL, Downey GP. Mechanics of stimulated neutrophils: cell stiffening induces retention in capillaries. Science 1989;245:183-186.

9. Yoshida K, Kondo R, Wang Q, Doerschuk CM. Neutrophil cytoskeletal rearrangements during capillary sequestration in bacterial pneumonia in rats. Am J Respir Crit Care Med 2006;174: 689-698.

10. Doerschuk CM. The role of CD18-mediated adhesion in neutrophil sequestration induced by infusion of activated plasma in rabbits. $A m$ J Respir Cell Mol Biol 1992;7:140-148.

11. Mizgerd JP, Horwitz BH, Quillen HC, Scott ML, Doerschuk CM. Effects of CD18 deficiency on the emigration of murine neutrophils during pneumonia. J Immunol 1999;163:995-999.

12. Kumasaka T, Doyle NA, Quinlan WM, Graham L, Doerschuk CM. Role of CD 11/CD 18 in neutrophil emigration during acute and recurrent Pseudomonas aeruginosa-induced pneumonia in rabbits. $\mathrm{Am} \mathrm{J}$ Pathol 1996;148:1297-1305.

13. Moser M, Bauer M, Schmid S, Ruppert R, Schmidt S, Sixt M, Wang HV, Sperandio M, Fässler R. Kindlin-3 is required for $\beta 2$ integrinmediated leukocyte adhesion to endothelial cells. Nat Med 2009;15: 300-305.

14. Lefort CT, Rossaint J, Moser M, Petrich BG, Zarbock A, Monkley SJ, Critchley DR, Ginsberg MH, Fässler R, Ley K. Distinct roles for talin-1 and kindlin-3 in LFA-1 extension and affinity regulation. Blood 2012; 119:4275-4282.

15. Lefort CT, Ley K. Neutrophil arrest by LFA-1 activation. Front Immunol 2012;3:157. 
16. Shimaoka M, Salas A, Yang W, Weitz-Schmidt G, Springer TA. Small molecule integrin antagonists that bind to the $\beta 2$ subunit I-like domain and activate signals in one direction and block them in the other. Immunity 2003;19:391-402.

17. Zarbock A, Lowell CA, Ley K. Spleen tyrosine kinase Syk is necessary for E-selectin-induced $\alpha_{\llcorner} \beta_{2}$ integrin-mediated rolling on intercellular adhesion molecule-1. Immunity 2007;26: 773-783.

18. Salas A, Shimaoka M, Kogan AN, Harwood C, von Andrian UH, Springer TA. Rolling adhesion through an extended conformation of integrin $\alpha_{\llcorner} \beta_{2}$ and relation to $\alpha$ I and $\beta$ I-like domain interaction. Immunity 2004;20:393-406.

19. Petrich BG, Marchese P, Ruggeri ZM, Spiess S, Weichert RA, Ye F, Tiedt R, Skoda RC, Monkley SJ, Critchley DR, et al. Talin is required for integrin-mediated platelet function in hemostasis and thrombosis. J Exp Med 2007;204:3103-3111.

20. Klapproth S, Moretti FA, Zeiler M, Ruppert R, Breithaupt U, Mueller S, Haas R, Mann M, Sperandio M, Fässler R, et al. Minimal amounts of kindlin-3 suffice for basal platelet and leukocyte functions in mice. Blood 2015;126:2592-2600.

21. Kühn R, Schwenk F, Aguet M, Rajewsky K. Inducible gene targeting in mice. Science 1995;269:1427-1429.

22. Liu PV. The roles of various fractions of Pseudomonas aeruginosa in its pathogenesis. 3. Identity of the lethal toxins produced in vitro and in vivo. $J$ Infect Dis 1966;116:481-489.

23. Reutershan J, Basit A, Galkina EV, Ley K. Sequential recruitment of neutrophils into lung and bronchoalveolar lavage fluid in LPSinduced acute lung injury. Am J Physiol Lung Cell Mol Physiol 2005; 289:L807-L815.

24. Qin L, Quinlan WM, Doyle NA, Graham L, Sligh JE, Takei F, Beaudet AL, Doerschuk CM. The roles of CD11/CD18 and ICAM-1 in acute Pseudomonas aeruginosa-induced pneumonia in mice. $\mathrm{J}$ Immunol 1996;157:5016-5021.

25. Kunkel EJ, Jung U, Bullard DC, Norman KE, Wolitzky BA, Vestweber D, Beaudet AL, Ley K. Absence of trauma-induced leukocyte rolling in mice deficient in both $\mathrm{P}$-selectin and intercellular adhesion molecule 1. J Exp Med 1996;183:57-65.

26. Wang GG, Calvo KR, Pasillas MP, Sykes DB, Häcker H, Kamps MP. Quantitative production of macrophages or neutrophils ex vivo using conditional Hoxb8. Nat Methods 2006;3: 287-293.

27. Miksa M, Komura H, Wu R, Shah KG, Wang P. A novel method to determine the engulfment of apoptotic cells by macrophages using pHrodo succinimidyl ester. J Immunol Methods 2009;342: 71-77.

28. Anderson DC, Springer TA. Leukocyte adhesion deficiency: an inherited defect in the Mac-1, LFA-1, and p150,95 glycoproteins. Annu Rev Med 1987;38:175-194.

29. Svensson L, Howarth K, McDowall A, Patzak I, Evans R, Ussar S, Moser M, Metin A, Fried M, Tomlinson I, et al. Leukocyte adhesion deficiency-III is caused by mutations in KINDLIN3 affecting integrin activation. Nat Med 2009;15:306-312.
30. Malinin NL, Zhang L, Choi J, Ciocea A, Razorenova O, Ma YQ, Podrez EA, Tosi M, Lennon DP, Caplan Al, et al. A point mutation in KINDLIN3 ablates activation of three integrin subfamilies in humans. Nat Med 2009;15:313-318.

31. Kuijpers TW, Van Lier RA, Hamann D, de Boer M, Thung LY, Weening RS, Verhoeven AJ, Roos D. Leukocyte adhesion deficiency type 1 $(L A D-1) / v a r i a n t$. A novel immunodeficiency syndrome characterized by dysfunctional $\beta 2$ integrins. J Clin Invest 1997;100:1725-1733.

32. Mizgerd JP. Molecular mechanisms of neutrophil recruitment elicited by bacteria in the lungs. Semin Immunol 2002;14:123-132.

33. Moser M, Legate KR, Zent R, Fässler R. The tail of integrins, talin, and kindlins. Science 2009;324:895-899.

34. Semmrich M, Smith A, Feterowski C, Beer S, Engelhardt B, Busch DH, Bartsch B, Laschinger M, Hogg N, Pfeffer K, et al. Importance of integrin LFA-1 deactivation for the generation of immune responses. $J$ Exp Med 2005;201:1987-1998.

35. Morin NA, Oakes PW, Hyun YM, Lee D, Chin YE, King MR, Springer TA, Shimaoka M, Tang JX, Reichner JS, et al. Nonmuscle myosin heavy chain IIA mediates integrin LFA-1 de-adhesion during T lymphocyte migration. J Exp Med 2008;205:195-205. [Published erratum appears in J Exp Med 205:993.\}

36. Koh AY, Priebe GP, Ray C, Van Rooijen N, Pier GB. Inescapable need for neutrophils as mediators of cellular innate immunity to acute Pseudomonas aeruginosa pneumonia. Infect Immun 2009;77:5300-5310.

37. Sun L, Guo RF, Newstead MW, Standiford TJ, Macariola DR, Shanley TP. Effect of IL-10 on neutrophil recruitment and survival after Pseudomonas aeruginosa challenge. Am J Respir Cell Mol Biol 2009;41:76-84.

38. Tsai WC, Strieter RM, Mehrad B, Newstead MW, Zeng X, Standiford TJ. CXC chemokine receptor CXCR2 is essential for protective innate host response in murine Pseudomonas aeruginosa pneumonia. Infect Immun 2000;68:4289-4296.

39. Bodey GP. Pseudomonas aeruginosa infections in cancer patients: have they gone away? Curr Opin Infect Dis 2001;14:403-407.

40. Ramphal R. Changes in the etiology of bacteremia in febrile neutropenic patients and the susceptibilities of the currently isolated pathogens. Clin Infect Dis 2004;39:S25-S31.

41. Oishi K, Sonoda F, Iwagaki A, Ponglertnapagorn P, Watanabe K, Nagatake T, Siadak A, Pollack M, Matsumoto K. Therapeutic effects of a human antiflagella monoclonal antibody in a neutropenic murine model of Pseudomonas aeruginosa pneumonia. Antimicrob Agents Chemother 1993;37:164-170.

42. Scarff JM, Goldberg JB. Vaccination against Pseudomonas aeruginosa pneumonia in immunocompromised mice. Clin Vaccine Immunol 2008;15:367-375.

43. Yamamoto K, Ahyi AN, Pepper-Cunningham ZA, Ferrari JD, Wilson AA, Jones MR, Quinton LJ, Mizgerd JP. Roles of lung epithelium in neutrophil recruitment during pneumococcal pneumonia. $A m \mathrm{~J}$ Respir Cell Mol Biol 2014;50:253-262.

44. Grommes J, Soehnlein O. Contribution of neutrophils to acute lung injury. Mol Med 2011;17:293-307.

45. Williams BJ, Dehnbostel J, Blackwell TS. Pseudomonas aeruginosa: host defence in lung diseases. Respirology 2010;15:1037-1056. 